University of Nebraska - Lincoln

DigitalCommons@University of Nebraska - Lincoln

1997

\title{
Inner-Shell Excitation Spectroscopy of closo-Carboranes
}

Adam P. Hitchcock

Stephen G. Urquhart

Alex T. Wen

A. L. David Kilcoyne

Tolek Tyliszczak

See next page for additional authors

Follow this and additional works at: https://digitalcommons.unl.edu/physicsdowben

Part of the Physics Commons

Hitchcock, Adam P.; Urquhart, Stephen G.; Wen, Alex T.; Kilcoyne, A. L. David; Tyliszczak, Tolek; Rühl, Eckart; Kosugi, Nobuhiro; Bozek, John D.; Spencer, James T.; Mcllroy, David N.; and Dowben, Peter A., "Inner-Shell Excitation Spectroscopy of closo-Carboranes" (1997). Peter Dowben Publications. 228. https://digitalcommons.unl.edu/physicsdowben/228

This Article is brought to you for free and open access by the Research Papers in Physics and Astronomy at DigitalCommons@University of Nebraska - Lincoln. It has been accepted for inclusion in Peter Dowben Publications by an authorized administrator of DigitalCommons@University of Nebraska - Lincoln. 


\section{Authors}

Adam P. Hitchcock, Stephen G. Urquhart, Alex T. Wen, A. L. David Kilcoyne, Tolek Tyliszczak, Eckart Rühl, Nobuhiro Kosugi, John D. Bozek, James T. Spencer, David N. Mcllroy, and Peter A. Dowben 


\title{
Inner-Shell Excitation Spectroscopy of closo-Carboranes
}

\author{
Adam P. Hitchcock,,,,$+ *$ Stephen G. Urquhart, ${ }^{\dagger}$ Alex T. Wen, ${ }^{\dagger}$ A. L. David Kilcoyne ${ }^{\dagger}$

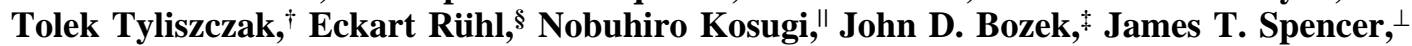 \\ David N. McIlroy, ${ }^{\nabla}$ and Peter A. Dowben ${ }^{\nabla, \perp}$ \\ Department of Chemistry, McMaster University, Hamilton, Ontario, Canada, L8S 4M1, Advanced Light \\ Source, Lawrence Berkeley Laboratory, Berkeley, California 91420, Center for Materials Research and \\ Analysis, Department of Physics, University of Nebraska-Lincoln, Lincoln, Nebraska 68588-0111, Institut für \\ Physik, Johannes Gutenberg-Universität Mainz, D-55099 Mainz, Germany, Institute for Molecular Science, \\ Myodaiji, Okazaki 444, Japan, and W. M. Keck Center for Molecular Electronics and the Department of \\ Chemistry, Syracuse, University, Syracuse, New York 13244-4100
}

Received: July 29, 1996; In Final Form: November 7, $1996^{\otimes}$

\begin{abstract}
Oscillator strength spectra in the region of $\mathrm{B} 1 \mathrm{~s}$ and $\mathrm{C} 1 \mathrm{~s}$ excitation of three isomeric carborane cage compounds [closo-1,2-orthocarborane, closo-1,7-metacarborane, closo-1,12-paracarborane $\left(\mathrm{C}_{2} \mathrm{~B}_{10} \mathrm{H}_{12}\right)$ ] have been derived from inner-shell electron energy loss spectra (ISEELS) recorded under electric dipole-scattering conditions. Total ion yield spectra recorded at high resolution with synchrotron radiation are also reported. The spectral features are assigned on the basis of comparisons with spectral predictions derived from the results of ab initio and semiempirical (extended Hückel) molecular orbital calculations. The isomeric and core level variations in the discrete core excitations are related to changes in orbital symmetries as well as variations in electron localization in these isomers. The ionization efficiency in the region of the B $1 \mathrm{~s}$ and $\mathrm{C}$ 1s edges is derived.
\end{abstract}

\section{Introduction}

Borane and carborane cage compounds have potential applications as source compounds for chemical vapor deposition (CVD) of a wide variety of thin film materials including variable band gap boron carbide thin film semiconductors ${ }^{1-4}$ and borondoped silicon..$^{5-11}$ Boron carbide devices (of $\mathrm{B}_{5} \mathrm{C}$ stoichiometry) have now been fabricated using orthocarborane as the source material. $3,4,12$ Since many of the borane and carborane molecules used as CVD sources contain only the elements of interest, these molecules can be decomposed with very short wavelength radiation to form high-quality films, thus facilitating selective area deposition with excellent spatial resolution. To demonstrate this potential, devices have been fabricated using synchrotron radiation-induced chemical vapor deposition. ${ }^{3,12}$ This type of device processing is not generally possible with most CVD source molecules-indeed, ref 3 reports the first such example. Although devices have now been fabricated with soft $\mathrm{X}$-ray radiation, ${ }^{3}$ the growth of semiconducting boron carbide is not understood. ${ }^{12}$ Characterizing the electronic excitations and the preferred decomposition pathways is critical to the elucidation of the mechanisms of the electron- and photoninduced decomposition. ${ }^{12}$ Currently, closo-1,2-dicarbadodecaborane is one of three potential carborane source molecules whose synchrotron radiation-assisted decomposition has been investigated. ${ }^{12}$ Although this boron carbide source molecule has been studied the most extensively, the details of the decomposition are still relatively poorly understood. $3,12,13$

The building block of the rhombohedral crystalline boron carbides, $\mathrm{B}_{12-x} \mathrm{C}_{x}, 0 \leq x \leq 2$, is a $\mathrm{B}_{12}$ icosahedral unit with carbon substitution. ${ }^{12,14,15}$ The changes with different substitution patterns in the electronic structure of boron carbide

\footnotetext{
$\dagger$ McMaster University.

¥ Lawrence Berkeley Laboratory.

§ Johannes Gutenberg-Universität Mainz. Present address: Fachbereich Physik, Universität Osnabrück, D-49069 Osnabrück, Germany.

"Institute for Molecular Science.

$\perp$ Syracuse University.

$\nabla$ University of Nebraska-Lincoln.

${ }^{\otimes}$ Abstract published in Advance ACS Abstracts, February 15, 1997.
}

\section{SCHEME 1}

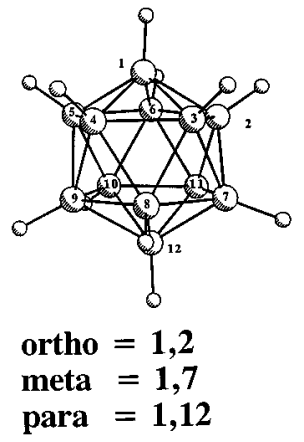

icosahedra have been considered a controlling factor in their electronic properties. Since the carboranes are hydrogenterminated $\mathrm{B}_{10} \mathrm{C}_{2}$ icosahedra, it is reasonable to use the electronic structure and spectroscopy of these species to gain insight into the properties of the semiconducting polytypes of the boroncarbon alloys from which devices have been made. ${ }^{1-4}$

This paper reports the B 1s and C 1s spectra of orthocarboane, metacarborane, and paracarborane recorded with both dipoleregime electron impact and synchrotron radiation. In an accompanying paper ${ }^{16}$ the ionic fragmentation of the $\mathrm{B} 1 \mathrm{~s}$ and $\mathrm{C} 1 \mathrm{~s}$ excited and ionized states of these three species is reported and compared to electron impact-induced fragmentation. The B 1s and C 1s excitation spectra of orthocarborane have been reported earlier and partially interpreted with the aid of semiempirical calculations. ${ }^{17}$ To our knowledge, this is the first presentation of the inner-shell spectroscopy of the metacarborane and paracarborane species. The structures of these molecules are presented in Scheme 1. Because there are relatively subtle changes in the spectra of these isomers, we have relied on highlevel ab initio calculations of the ground and core excited states to provide a detailed picture of the electronic structure of these species in the gas phase and to form a basis for interpreting the experimental spectra. In addition, the experimental and ab initio results are compared to spectral predictions based on semiempirical extended Hückel calculations carried out within the 
equivalent core approximation. This comparison is used to investigate the merit of extended Hückel molecular orbital (EHMO) calculations in treating core excitation of systems with extensive electronic delocalization.

The nature of the electronic delocalization in these compounds is of considerable practical interest, since it may be connected to conduction mechanisms of the closely related icosahedral boron-carbon alloys. Calculations ${ }^{18}$ show that the charge in similar icosahedra $\left(\mathrm{B}_{11} \mathrm{C}\right)$ is delocalized for both $\mathrm{B}_{11} \mathrm{C}^{-}$and $\mathrm{B}_{11} \mathrm{C}^{+}$ions. The consequences for charge delocalization in the free molecular icosahedra as well as in the icosahedral solids are very important. This means that for the free molecular species, the cluster remains fairly stable despite charge-induced distortion. For the icosahedral solid, this charge delocalization suggests that charge occupies the icosahedra in the solid and conduction or electron transport occurs via hopping between adjacent icosahedra. ${ }^{18}$

In related studies, Ruckman, Strongin, and co-workers ${ }^{19}$ have recently investigated the B 1s spectrum of solid closo-1,2orthocarborane, while Green and co-workers have reported ab initio calculations within the $(Z+1)$ approximation of the $\mathrm{B}$ $1 \mathrm{~s}$ spectrum of orthocarborane $\mathrm{e}^{20}$ as well as calculations of the ground state of paracarborane..$^{21}$

\section{Experimental Section}

2.1. Materials. All solvents (tetrahydrofuran, pentane, and diethyl ether) were reagent grade or better and were distilled from the appropriate drying agents $(\mathrm{Na})$ under a dry nitrogen atmosphere prior to use. After drying, all organic solvents were degassed with a dry nitrogen stream and then by repeated freeze-thaw cycles, then stored in vacuo prior to use. Deuterated solvents were used as received and, after degassing, were stored over $4 \AA$ molecular sieves prior to use. The commercially available anhydrous chemicals were used either as received or purified by the method indicated and, where possible, were stored over $4 \AA$ molecular sieves prior to use. The identity and purity of all compounds were determined by NMR, IR, and mass spectral measurements and compared with literature values.

2.2. Physical Measurements. The inner-shell electron energy loss spectrometer and operating techniques have been described previously. ${ }^{22}$ The spectra were obtained by inelastic electron scattering with a high velocity incident electron beam $\left(E_{\mathrm{o}}=2.5 \mathrm{keV}\right.$ plus the energy loss), small scattering angle $\left(<2^{\circ}\right)$, and $0.7 \mathrm{eV}$ fwhm overall energy resolution. To achieve adequate vapor pressure in the collision region of the spectrometer for the lower volatility ortho and meta species, the sample cell, inlet line, and collision chamber were gently heated to about $50{ }^{\circ} \mathrm{C}$. Heating was not required to run the para isomer. The B 1s and C 1s spectra were converted to absolute optical oscillator strengths ( $f$ values) using a procedure described and tested previously. ${ }^{23}$

The photoexcitation spectra were recorded using both total electron yield and total ion yield detection at beam-line 9.0.1 of the Advanced Light Source. ${ }^{24}$ The beam-line consists of a spherical grating monochromator illuminated by the radiation from an undulator. The apparatus is described in greater detail in the accompanying paper on the ionic fragmentation. ${ }^{16}$ Briefly, a time-of-flight tube with a channel plate detector and a channeltron are mounted on opposite sides of the intersection of the focused $(<0.1 \mathrm{~mm}$ diameter) photon beam and an effusive gas jet. A high extraction field $\left(>250 \mathrm{~V} \mathrm{~cm}^{-1}\right)$ was used for these measurements. Slit widths of less than $20 \mu \mathrm{m}$ were used, providing a spectral resolution better than $0.1 \mathrm{eV}$. The undulator gap was optimized at each photon energy during spectral acquisition. The spectra were normalized to the incident flux that was measured from the current at a Au mesh placed after the interaction region.
2.3. Theoretical Calculations. 2.3.1. Ab Initio Calculations. The ground-state geometries of the three isomers were optimized using energy minimization with the standard 4-31G* basis set. Six structural parameters were optimized for paracarborane and 20 for metacarborane and orthocarborane. The results are shown in Table 1 . In general they are close to the experimental geometries. ${ }^{25}$ The symmetry is $D_{5 d}$ for para and $C_{2 v}$ for meta and ortho. In all core excited-state calculations the ground-state geometries are used.

The core-ionized and core excited-state energies and the oscillator strengths for the core excitation transitions were obtained by ab initio SCF calculations with explicit consideration of the core hole ${ }^{26}$ and with the localized core-hole picture. We used a minimal basis (MB) set taken from (73/7) and (73/6) contracted Gaussian-type functions for $\mathrm{B}$ and $\mathrm{C}$ and (6) for $\mathrm{H}$ of Huzinaga et al. ${ }^{27}$ For comparison extended basis (EB) sets were also used for paracarborane. In that calculation the contraction scheme was $\left(4111111 / 31111 / 1^{*}\right)$ for atoms with a core hole, (621/41) for atoms with no core hole, and (41) for H. Furthermore, ab initio SCF calculations based on the equivalent ionic core virtual orbital (EICVOM) model $^{28}$ and the minimal basis set were performed for comparison. In that case the relative intensities are evaluated by using $2 p$ atomic orbital coefficients of the equivalent $(Z+1)$ atom.

The core excited states were obtained with the improved virtual orbital (IVO) method. ${ }^{29}$ The relaxed HF potential is essential in accurately considering large electronic reorganization upon inner-shell hole creation. Therefore, the IVO method based on the relaxed HF potential is superior to the method using the ground-state orbitals. This method is excellent in predicting term values and intensities of core-to-Rydberg excited states $^{30,31}$ and is used as a first-order approximation for coreto-valence excited states. ${ }^{32}$ The calculations were performed using an original ab initio code called GSCF3. ${ }^{33}$

2.3.2. Extended Hückel Calculations. Extended Hückel (EHMO) calculations were carried out on these molecules using standard methods. ${ }^{34}$ The default Hückel parameters were used. The geometries used were those determined by ab initio geometry optimization using $4-31 \mathrm{G}^{*}$ basis sets as shown in Table 1. To facilitate interpretation of the B 1 s and C 1 s spectra, EHMO calculations relevant to the core excited states were carried out using the equivalent ionic core virtual orbital (EICVOM, or $Z+1$ ) model. ${ }^{28}$ Relative intensities were derived from the EICVOM $(Z+1)$ calculations using the approach documented in our earlier work. ${ }^{17}$ Gaussian line widths used in generating simulated spectra from both the EHMO and ab initio results were $5 \mathrm{eV}$ fwhm for orbitals of eigenvalue $\epsilon$ above $2 \mathrm{eV}, 3 \mathrm{eV}$ for $-1<\epsilon<2,1 \mathrm{eV}$ for $-15<\epsilon<-1 \mathrm{eV}$.

\section{Results and Discussion}

3.1. Presentation of $B$ 1s and C 1s Spectra. The B 1s oscillator strength spectra of the isomeric carboranes derived from the ISEELS are presented in Figure 1, while the discrete regions of the high-resolution total ion yield (TIY) spectra are presented as an insert. The energies, term values, and proposed assignments are listed in Table 2. The B 1s spectra of all isomers are dominated by the strong resonance centered near $192 \mathrm{eV}$. At higher resolution isomer-dependent fine structure is visible within this band (see insert of Figure 1), indicating that the B 1s spectra are sensitive to differences in the electronic structure of the isomers. For orthocarborane the $192 \mathrm{eV}$ band was assigned earlier to overlap of excitations to the $10 \mathrm{a}^{\prime \prime}$ and $17 \mathrm{a}^{\prime}$ from all the B 1 s orbitals. ${ }^{17}$ The other prominent B $1 \mathrm{~s}$ spectral features are two broad bands located in the B 1s continuum (197 and $202 \mathrm{eV}$ ). In the para and meta isomers there is a distinct shoulder at $195 \mathrm{eV}$ that is not seen in 
TABLE 1: Geometries Predicted from ab Initio Hartree-Fock Calculations with the 4-31G* Basis Set

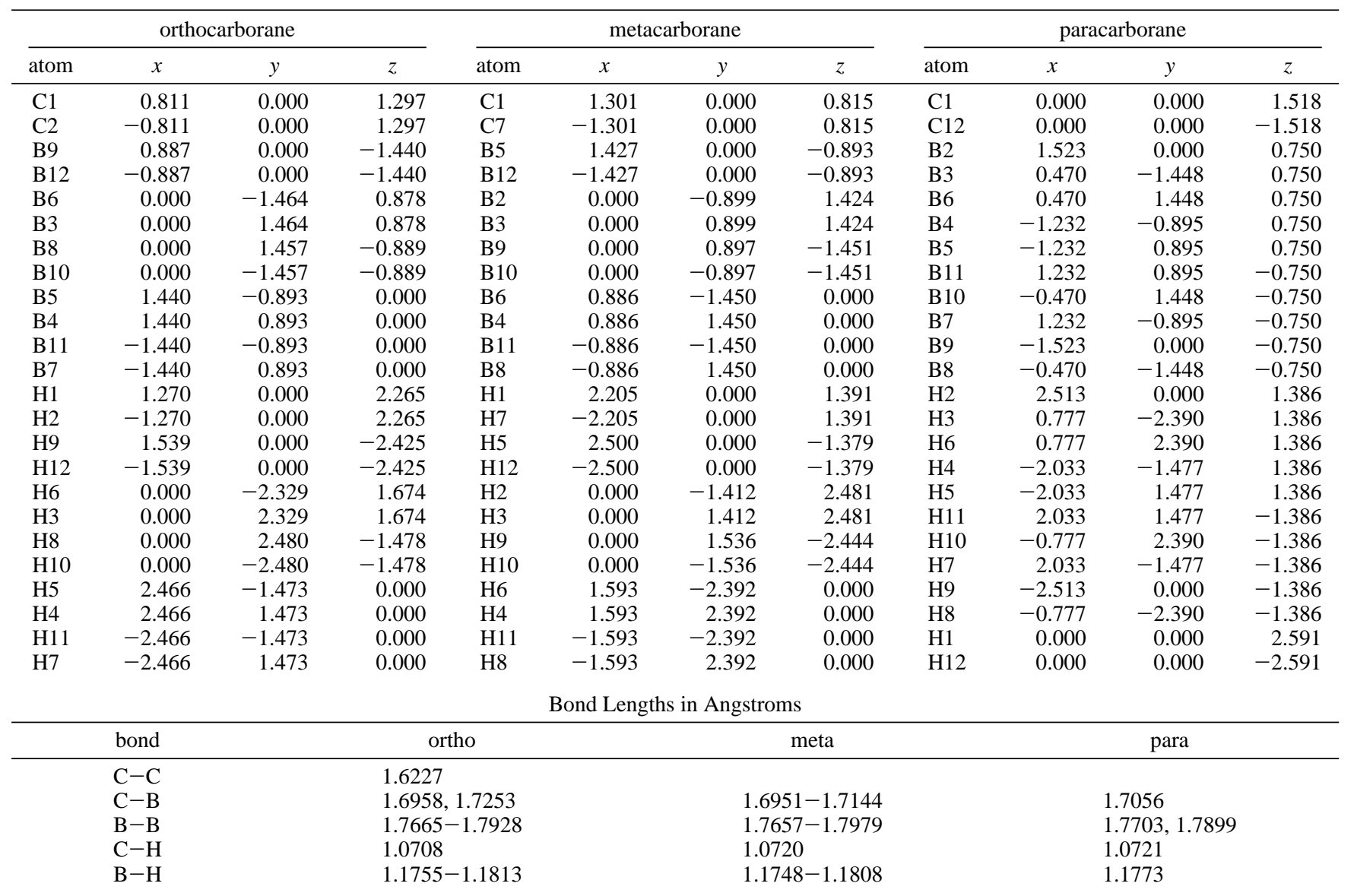

orthocarborane. The higher energy continuum transitions in orthocarborane were attributed to excitations to antibonding $\sigma^{*}$ orbitals of unspecified symmetry. ${ }^{17}$ Clearly, a more sophisticated treatment of the electronic structure and spectroscopy is required to explain the significant but subtle isomeric variations in the B 1s spectra.

The $\mathrm{C}$ 1s oscillator strength spectra of the isomeric carboranes derived from the ISEELS are presented in Figure 2, while the discrete regions of the high-resolution total ion yield (TIY) spectra are presented as an insert. The energies, term values, and proposed assignments are listed in Table 3. As with the B 1s spectra, there are subtle but significant differences in the $C$ $1 \mathrm{~s}$ spectra of these isomers. The intense lowest energy feature in the 287.9-288.7 eV range occurs at different energies for each of the three isomers. In addition, the spectra also differ in shape above $290 \mathrm{eV}$ excitation energy. Such distinct spectral changes cannot be interpreted solely on the basis of an intuitive assignment as has been possible in the case of many organic molecules. Carborane molecules are known to have delocalized orbital structure. ${ }^{18-20}$ This makes assignment of the inner-shell absorption features more difficult than for small molecules. As a consequence, we have based the spectral assignments on highlevel ab initio calculations. At the same time, since semiempirical calculations have proven to be of considerable use in recent core excitation studies, we have also compared the EHMO results to the ab initio and experimental results in order to evaluate the utility of the EHMO method in these systems.

3.2. Paracarborane: Basis Set Dependence of ab Initio Calculations. Figure 3 presents the $\mathrm{C} 1 \mathrm{~s}$ and $\mathrm{B}$ 1s spectra of paracarborane generated from ab initio calculations using three different levels of approximation: a minimal basis, equivalent core $(Z+1)$ treatment $(Z+1-\mathrm{MB})$; the improved virtual orbital method using a minimal basis set (IVO-MB); the improved virtual orbital method using an extended basis set (IVO-EB). The experimental TIY spectrum is also included in Figure 3 for comparison. Although all three treatments provide useful insight into the spectra, the IVO-EB gives the closest match to experimental results, at least for the discrete transitions. Resonances in the continuum that are regarded as excitations to antibonding orbitals are approximated within the framework of the minimal basis set. ${ }^{35}$ On the other hand, in extended basis (EB) calculations some basis functions are partly used for description of the continuum, and thus, it is difficult to describe resonance states due to fortuitous contributions from the continuum. This difficulty can be removed by the $L^{2}$ method. ${ }^{36}$ Since this approach was not used, Figure 3 shows only boundstate resonances for the IVO-EB results. It is noted that in the extended basis calculation some contributions from Rydberg states are also included, with the attribution of Rydberg or valence character being based on mean radial extent of the wave function for the excited electron.

To facilitate comparison with experiment, the first peak in each calculation has been aligned with the first experimental peak. This has involved the following shifts in the calculated energies: for $\mathrm{B} 1 \mathrm{~s},-2.0 \mathrm{eV}$ for IVO-EB, $+2.0 \mathrm{eV}$ for IVO$\mathrm{MB}$, and $+1.4 \mathrm{eV})$ for $(Z+1)-\mathrm{MB}$ (after the $(Z+1)-\mathrm{MB}$ eigenvalue zero is aligned at the experimental $\mathrm{B}$ 1s IP; for $\mathrm{C}$ $1 \mathrm{~s},-1.8 \mathrm{eV}$ for IVO-EB, $+1.8 \mathrm{eV}$ for IVO-MB, and $+1.5 \mathrm{eV}$ for $(Z+1)$-MB (after $\mathrm{C} 1 \mathrm{~s}$ IP alignment). The core ionized state obtained with the minimal basis set is relatively poorly described because of the inability of the IVO-MB approach to reproduce large orbital reorganization upon core-hole creation. However, the core excited state is relatively well described with the IVO-MB approach because the excited valence electron provides core-hole screening. Because of these differing relative errors, the IVO-MB calculation predicts larger term values for bound state resonances than are observed experimentally. On the other hand, the core ionized state is well described with the extended basis set, whereas the core excited state is not treated as well. Therefore, the IVO-EB calculation predicts smaller 


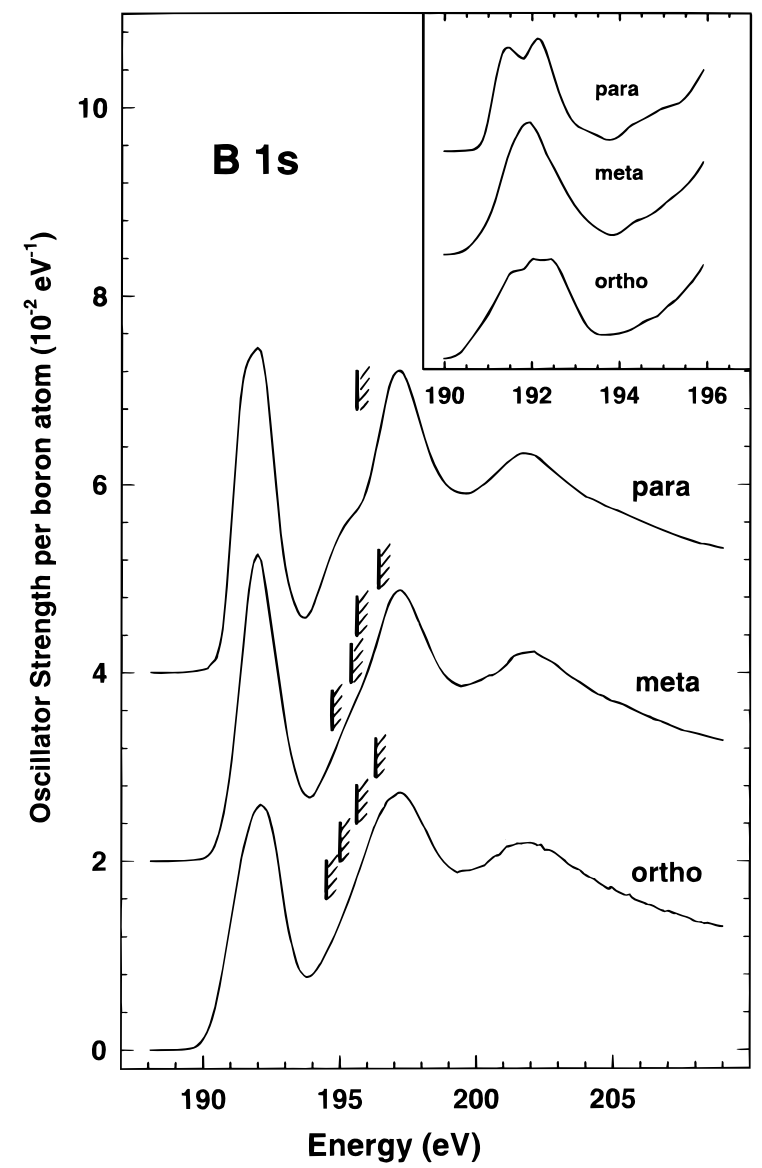

Figure 1. B 1s oscillator strength spectra for the isomeric carboranes derived from electron energy loss spectra recorded with $2.5 \mathrm{keV}$ final electron energy, $2^{\circ}$ scattering angle, and $0.7 \mathrm{eV}$ fwhm resolution. The insert plots the low-energy region of the total ion yield spectra recorded with synchrotron radiation at the Advanced Light Source with $<0.1$ $\mathrm{eV}$ fwhm resolution. The hatched lines indicate the ionization limits as determined by XPS ${ }^{38}$ (ortho, meta) or estimated (para).

term values than those observed experimentally. Given that the IVO-MB and IVO-EB calculations are computing the full core excitation energy, achieving a match with the experimental excitation energies to within $2 \mathrm{eV}$ or less (i.e., $\sim 1 \%$ ) is quite impressive.

The ab initio results predict two main contributions to the second feature of the B $1 \mathrm{~s}$ spectrum of paracarborane, that corresponding to the peak at $192.4 \mathrm{eV}$. However, the relative intensities of these two components depend on the basis set and computational method used. In the $\mathrm{C} 1 \mathrm{~s}$ spectrum of paracarborane (Figure 3b) the weak second peak at $289.4 \mathrm{eV}$ is reproduced by the calculations, but it is predicted to be relatively higher in energy than observed experimentally, especially in the IVO-MB calculation. Similar disagreement was observed in the minimal basis set calculations of orthocarborane and metacarborane. The very weak structure predicted at $288.7 \mathrm{eV}$ in the extended basis set calculation of the $\mathrm{C} 1 \mathrm{~s}$ spectrum of paracarborane is of Rydberg character, based on its orbital size.

3.3. Paracarborane: Exploring MO Character and Relationship to Core Spectra. A detailed consideration of the characters of the MOs in the ground and core excited states is helpful to gain further insight into the assignments of the discrete spectral features. Figure 4 compares the shapes of the four lowest energy MOs in the ground state, B 1s and C 1s excited states of paracarborane. In the ground state the lowest unoccupied MOs (LUMO) are degenerate as shown in Figure 4a. Note that only the upper six atoms $(1-6)$ are shown in Figure 4. There are similar contributions from the lower six atoms (7-12) in the B 1s and C 1s excited states as well as the ground

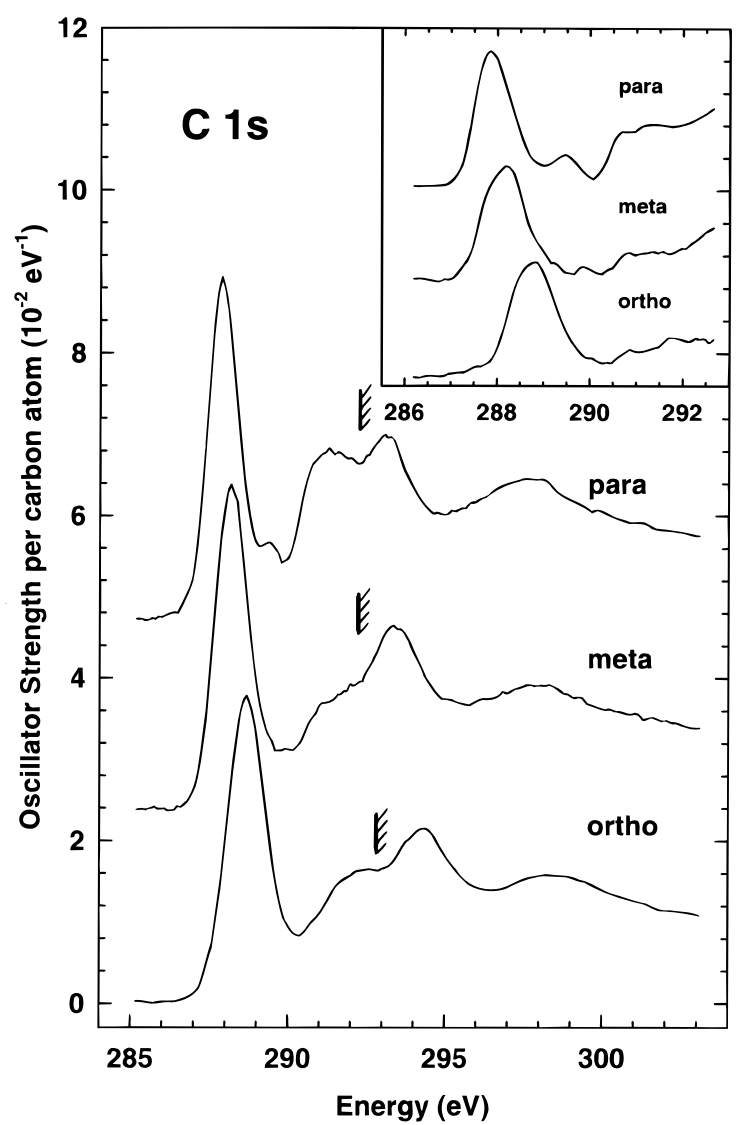

Figure 2. C 1s oscillator strength spectra of the isomeric carboranes derived from ISEELS (see caption to Figure 1 for experimental details). The insert shows the low-energy region of the high-resolution $(<0.1$ $\mathrm{eV}$ fwhm) TIY spectra. IPs are from XPS ${ }^{38}$ (ortho, meta) or estimated (para).

state. Any orthogonal transformation is possible between degenerate MOs. We have chosen to transform the MOs to maintain a one-to-one correspondence with those that are produced by symmetry breaking upon creating a localized core hole at $\mathrm{B}_{2}$ (for atom numbering, see the plot of the $\mathrm{e}_{1 \mathrm{~g}}(y)$ orbital for the ground state in Figure 4a). The two LUMOs are labeled $\mathrm{e}_{\mathrm{gg}}(x)$ and $\mathrm{e}_{\mathrm{lg}}(y)$, consistent with the overall MO symmetry and the spatial orientation of the $2 p$ orbital components at $C_{1}$. In the $\mathrm{e}_{1 \mathrm{~g}}(x)$ LUMO there is negligible contribution from $\mathrm{B}_{2}$, and in the $\mathrm{e}_{1 \mathrm{~g}}(y)$ LUMO there is little contribution from $\mathrm{B}_{3}$ and $\mathrm{B}_{6}$. On the other hand, in the second lowest unoccupied MO (LUMO +1$)$, which is also degenerate, there is no contribution from the carbon atoms. The two (LUMO + 1) components are labeled $\mathrm{e}_{2 \mathrm{~g}}(x z)$ and $\mathrm{e}_{2 \mathrm{~g}}(y)$, consistent with the overall $\mathrm{MO}$ symmetry and the spatial orientation of the $2 \mathrm{p}$ orbital components at $\mathrm{B}_{2}$. In the (LUMO + 1) $\mathrm{e}_{2 \mathrm{~g}}(x z)$ there is a large contribution from $\mathrm{B}_{2} 2 \mathrm{p}_{x}$.

In the $\mathrm{C} 1 \mathrm{~s}$ excited state (Figure $4 \mathrm{~b}$ ) the symmetry is reduced to $C_{5 v}$ so that the lowest excited MO (C-LEMO) and second lowest excited MO $(\mathrm{C}$-LEMO +1$)$ are still degenerate. The molecular orbitals are very similar to those for the ground state. Transitions from the $\mathrm{C} 1 \mathrm{~s}$ to the $(\mathrm{C}-\mathrm{LEMO}+1)$ orbital are symmetry forbidden as is reflected in the absence of any contribution of the $\mathrm{C} 2 \mathrm{p}$ orbitals to the $(\mathrm{C}-\mathrm{LEMO}+1)$. This is the reason the $\mathrm{C} 1 \mathrm{~s}$ spectrum of paracarborane is simpler than the corresponding B 1s spectrum. There is a weak absorption about $1.5 \mathrm{eV}$ higher energy than the first strong $\mathrm{C} 1 \mathrm{~s}$ resonance, which is assigned to excitation to another, higher energy unoccupied orbital. This excited orbital is dominated by a $\mathrm{C}$ $\operatorname{sp}(z)$ hybrid orbital and has antibonding character with five boron atoms as shown in Figure $4 d$. It has $\mathrm{a}_{1 \mathrm{~g}}(z)$ symmetry in the $D_{5 d}$ manifold. A similar, high-energy transition is predicted 
TABLE 2: Energies, Term Values, and Proposed Assignments for Features in the B 1s Spectra of Isomeric closo-Carboranes

\begin{tabular}{|c|c|c|c|c|c|c|c|c|c|c|}
\hline \multicolumn{11}{|c|}{ closo-1,2-Orthocarborane $^{a}$} \\
\hline \multirow[b]{2}{*}{ no. } & \multicolumn{2}{|c|}{ energy $(\mathrm{eV})$} & \multicolumn{4}{|c|}{ term value $(\mathrm{eV})$} & \multicolumn{4}{|c|}{ assignment $^{b}$} \\
\hline & EELS & TIY & $T_{9,12}$ & $T_{8,10}$ & $T_{4,5,7,11}$ & $T_{3,6}$ & 9,12 & 8,10 & $4,5,7,11$ & 3,6 \\
\hline $1 \mathrm{sh}$ & & 190.6 & 3.9 & & & & $10 a^{\prime \prime}$ & & & \\
\hline $2 \mathrm{sh}$ & 191.3 & 191.5 & 3.0 & 3.7 & & & $17 a^{\prime}$ & $10 a^{\prime \prime}$ & & \\
\hline 3 & $192.1^{(\mathrm{c})}$ & 192.1 & & 2.9 & 3.5 & 4.2 & & $17 a^{\prime}$ & $26 \mathrm{a}$ & $10 a^{\prime \prime}$ \\
\hline $4 \mathrm{sh}$ & & 192.5 & & & 3.1 & 3.9 & & & $27 \mathrm{a}$ & $17 a^{\prime}$ \\
\hline & & 194.6 & & & & & & & & \\
\hline $6 \mathrm{sh}$ & & 195.0 & & & & & & & & \\
\hline IP $(9,12)$ & $194.5^{d}$ & & & & & & IP & & & \\
\hline $\operatorname{IP}(8,10)$ & $195.0^{d}$ & & & & & & & IP & & \\
\hline $\operatorname{IP}(4,5,7,11)$ & $195.6^{d}$ & & & & & & & & IP & \\
\hline $\operatorname{IP}(3,6)$ & $196.3^{d}$ & & & & & & & & & IP \\
\hline 7 & 197.2 & 197.2 & -2 & -2 & -2 & & & & & \\
\hline 8 & 201.9 & 202.0 & -6 & -6 & -6 & & & & & \\
\hline \multicolumn{11}{|c|}{ closo-1,7-Metacarborane $^{a}$} \\
\hline & \multicolumn{2}{|c|}{ energy $(\mathrm{eV})$} & \multicolumn{4}{|c|}{ term value $(\mathrm{eV})$} & \multicolumn{4}{|c|}{ assignment $^{b}$} \\
\hline no. & EELS & TIY & $T_{9,10}$ & $T_{5,12}$ & $T_{4,6,8,11}$ & $T_{2,3}$ & 9,10 & 5,12 & $4,6,8,11$ & 2,3 \\
\hline $1 \mathrm{sh}$ & & 191.5 & 4.2 & & & & $17 a^{\prime}$ & & & \\
\hline $2 \mathrm{sh}$ & $191.88^{c}$ & 191.89 & 3.2 & 4.5 & 4.7 & 4.5 & $10 a^{\prime \prime}$ & $10 a^{\prime \prime}$ & $26 \mathrm{a}$ & $17 a^{\prime}$ \\
\hline 3 & & 194.4 & & 1.0 & 1.2 & 2.0 & & $17 a^{\prime}$ & $27 \mathrm{a}$ & $10 a^{\prime \prime}$ \\
\hline 4 & 195.0 & 195.0 & & & & & & & & \\
\hline IP $(9,10)$ & $194.7^{d}$ & & & & & & IP & & & \\
\hline $\operatorname{IP}(5,12)$ & $195.4^{d}$ & & & & & & & IP & & \\
\hline $\operatorname{IP}(4,6,8,11)$ & $195.6^{d}$ & & & & & & & & IP & \\
\hline $\operatorname{IP}(2,3)$ & $196.4^{(\mathbf{d})}$ & & & & & & & & & IP \\
\hline 5 & 197.2 & 197.1 & -2 & -2 & -2 & & & & & \\
\hline 6 & 202 & 202 & -6 & -6 & -6 & & & & & \\
\hline 7 (br) & 222 & & & & & & & & & \\
\hline \multicolumn{11}{|c|}{ closo-1,12-Paracarborane $^{a}$} \\
\hline \multicolumn{11}{|c|}{ energy $(\mathrm{eV})$} \\
\hline \multicolumn{2}{|l|}{ no. } & EELS & & \multicolumn{2}{|l|}{ TIY } & \multicolumn{2}{|c|}{$\mathrm{TV}(\mathrm{eV})$} & \multicolumn{3}{|c|}{ assignment $^{b}$} \\
\hline 1 & & 191.5 & & \multicolumn{2}{|l|}{191.45} & \multicolumn{2}{|c|}{5.1} & \multicolumn{3}{|c|}{$10 a^{\prime \prime}$} \\
\hline 2 & & $191.8^{c}$ & & \multicolumn{2}{|l|}{192.08} & \multicolumn{2}{|r|}{3.6} & & \multicolumn{2}{|c|}{$17 a^{\prime}$} \\
\hline $3 \mathrm{sh}$ & & & & \multicolumn{2}{|l|}{193.3} & \multicolumn{2}{|r|}{2.3} & & $18 \mathrm{a}^{\prime}$ & \\
\hline 4 & & & & 194.2 & & & 1.4 & & & \\
\hline 5 & & 195.0 & & 195.0 & & & 0.6 & & & \\
\hline IP (all) & & $195.6^{e}$ & & & & & & & IP & \\
\hline 7 & & 197.1 & & 197.1 & & & & & & \\
\hline 8 & & 201.7 & & 201.6 & & & 7 & & & \\
\hline 9 (br) & & 222 & & & & & 6 & & exaf & \\
\hline
\end{tabular}

${ }^{a}$ The labeling uses the atom numbering given in Scheme $1 .{ }^{b}$ Final level in reduced symmetry associated with localized-core hole (see text). ${ }^{c}$ Calibration: ortho $+7.52(8)$ eV relative to $\mathrm{S} 2 \mathrm{p}_{3 / 2} \rightarrow \mathrm{t}_{2 \mathrm{~g}}$ of $\mathrm{SF}_{6}$; meta $+7.49(8)$ eV relative to $\mathrm{S} 2 \mathrm{p}_{3 / 2} \rightarrow \mathrm{t}_{2 \mathrm{~g}}$ of $\mathrm{SF}_{6}$; para $-98.7 \mathrm{eV}$ from C $1 \mathrm{~s} \rightarrow$ $\pi^{*}$ of $\mathrm{CO}_{2} .{ }^{d}$ IPs from XPS. ${ }^{38}{ }^{e}$ IP estimated to be the same as boron with a $\mathrm{CB}_{4}$ local structure as in $\mathrm{B}_{4,5,7,11}$ in ortho and $\mathrm{B}_{4,6,8,11}$ in paracarborane.

in the IVO-MB calculation of all three carboranes. These correspond to experimental features that have term values of $2.0-2.3 \mathrm{eV}$. These excited states may have some Rydberg character. In fact, the IVO-EB calculation for paracarborane predicts a Rydberg contribution to the second peak.

The first resonance band in the B 1s spectrum of paracarborane has shoulders in addition to the two main peaks. The reason is that within the localized core-hole picture there are no degenerate excited states (see Figure 4c). As shown in Figure $4 \mathrm{c}$, the lowest excited MO (B-LEMO) $(y)$ is similar to the LUMO $\mathrm{e}_{\mathrm{g}}(y)$ in the ground state. Because the other LUMO $\mathrm{e}_{\mathrm{g}}(x)$ has little contribution from $\mathrm{B}_{2}$ in the ground state, excitation to this orbital is not stabilized by B 1s core hole creation. As shown in Figure 4c, this excited orbital corresponds to the third excited MO (B-LEMO + 2) $(x)$, and the excited state corresponds to the weak shoulder at $193.2 \mathrm{eV}$. Of the second LUMOs of the ground state, the (LUMO + 1) $\mathrm{e}_{2 \mathrm{~g}}(x z)$ is significantly deformed toward the $\mathrm{B}_{2}$ atom and is energetically stabilized. Thus, an excitation to the (B-LEMO +1$)(x z)$ orbital corresponds to the strong second peak. The fourth excited orbital $(\mathrm{B}-\mathrm{LEMO}+3)(y)$ is related to the $(\mathrm{LUMO}+1) \mathrm{e}_{2 \mathrm{~g}}(y)$ orbital, but it is relatively destabilized and has a reduced $\mathrm{B}_{2}$
$2 \mathrm{p}_{y}$ component. In other words, the $\mathrm{B}_{2} 2 \mathrm{p}_{y}$ component is "stolen" in order to stabilize the B-LEMO $(y)$. The net result is that the $\mathrm{B} 1 \mathrm{~s}$ spectrum is composed of two main peaks, which are related to excitations to the orbitals correlating with LUMO $\mathrm{e}_{1 \mathrm{~g}}(y)$ and $(\mathrm{LUMO}+1) \mathrm{e}_{2 \mathrm{~g}}(x z)$ in the ground state, and shoulder structures, which are related to the excitations to the LUMO $\mathrm{e}_{1 \mathrm{~g}}(x)$ and (LUMO +1$) \mathrm{e}_{2 \mathrm{~g}}(y)$ of the ground state, destabilized through mixing with (LUMO +1$) \mathrm{e}_{2 \mathrm{~g}}(x z)$ and LUMO $\mathrm{e}_{1 \mathrm{~g}}(y)$, respectively. Furthermore, the (LEMO +1$)(x z)$ and (LEMO $+2)(x)$ orbitals are mixed with each other because their energy difference is small and they have the same symmetry in the B $1 \mathrm{~s}$ excited state. The degree of admixture is heavily dependent on the approximations made in the calculation. The B $2 p$ contributions on the $\mathrm{B}_{2}$ atom in these two orbitals are also dependent on the details of the calculation with consequences on the predicted spectra as shown in Figure 3a. The third state related to $(\mathrm{LEMO}+2)$ is very weak in the IVO-MB calculation, whereas it is stronger than the second state in the $(Z+1)-\mathrm{MB}$ calculation. The very weak transition predicted at $192 \mathrm{eV}$ in the IVO-EB calculation is of Rydberg character.

The above discussion is based on the localized-core picture. The core hole is localized on one atom, but the excited orbitals 
TABLE 3: Energies, Term Values, and Proposed Assignments for Features in the C 1s Spectra of the Isomeric closo-Carboranes

\begin{tabular}{|c|c|c|c|c|}
\hline \multirow[b]{2}{*}{ no. } & \multicolumn{2}{|c|}{ energy $(\mathrm{eV})$} & \multirow[b]{2}{*}{ TV $(\mathrm{eV})$} & \multirow[b]{2}{*}{ assignment $^{a}$} \\
\hline & EELS & TIY & & \\
\hline \multicolumn{5}{|c|}{ closo-1,2-Orthocarborane } \\
\hline 1 & $288.65^{b}$ & 288.67 & 4.1 & $10 a^{\prime \prime}, 17 a^{\prime}$ \\
\hline $2 \mathrm{sh}$ & 290.8 & 290.77 & 2.0 & $3 p$ \\
\hline $3 \mathrm{sh}$ & 291.6 & 291.7 & 1.2 & $4 p$ \\
\hline 4 & 292.4 & 292.3 & 0.4 & Ryd \\
\hline IP & $292.8^{c}$ & & & \\
\hline 5 & 294.3 & 294.4 & -1.5 & $\sigma^{*}$ \\
\hline 6 & 298.4 & 298.6 & -7.6 & $\sigma^{*}$ \\
\hline 7 br & $304.3(5)$ & & & \\
\hline $8 \mathrm{br}$ & $321(1)$ & & -28 & $2 \mathrm{e}^{-}$or exafs \\
\hline \multicolumn{5}{|c|}{ closo-1,7-Metacarborane } \\
\hline $1 \mathrm{sh}$ & & 287.9 & 4.3 & $10 a^{\prime \prime}$ \\
\hline 2 & $288.16^{b}$ & 288.2 & 4.0 & $17 a^{\prime}$ \\
\hline 3 & & 289.9 & 2.3 & \\
\hline $4 \mathrm{sh}$ & 291.0 & 290.8 & 1.3 & $3 p$ \\
\hline $5 \mathrm{sh}$ & 291.8 & 291.4 & 0.8 & $4 p$ \\
\hline IP & $292.2^{c}$ & & & \\
\hline 6 & 293.3 & 293.5 & -1.1 & $\sigma^{*}$ \\
\hline 7 & 298.0 & 298.1 & -6.9 & $\sigma^{*}$ \\
\hline \multicolumn{5}{|c|}{ closo-1,12-Paracarborane } \\
\hline 1 & 287.9 & 287.85 & 4.3 & $10 \mathrm{e}_{1}$ \\
\hline 2 & $289.4^{b}$ & 289.5 & 2.7 & \\
\hline $3 \mathrm{sh}$ & 290.8 & 290.7 & 1.5 & $3 p$ \\
\hline $4 \mathrm{sh}$ & 291.3 & 291.3 & 0.9 & $4 \mathrm{p}$ \\
\hline IP & $292.2^{d}$ & & & \\
\hline 5 & 293.3 & 293.5 & -1.1 & $\sigma^{*}$ \\
\hline 6 & 297.6 & 297.9 & -6 & $\sigma^{*}$ \\
\hline
\end{tabular}

${ }^{a}$ Final level in reduced symmetry associated with localized-core hole (see text). ${ }^{b}$ Calibration: ortho $-2.1(1) \mathrm{eV}$; meta $-2.58(8) \mathrm{eV}$; para $-2.84(8) \mathrm{eV}$ relative to $\mathrm{CO}_{2} \pi^{*}(290.74 \mathrm{eV}) .{ }^{c}$ From XPS. ${ }^{38}{ }^{d}$ Estimated to be the same as that of metacarborane (see text).

are delocalized over all the atoms in the molecule. If the EICVOM $(Z+1)$ approximation provides a good description of the optical orbitals of the core excited states, it will represent the virtual orbitals whether they are localized or delocalized. In the delocalized-core picture, the MOs of the ground and all excited states belong to $D_{5 d}$ symmetry. Therefore, we have to consider core excited states by using degenerate $e_{1 g}$ and $e_{2 g}$ orbitals in the B 1s core excited states as well as in the ground state. However, it is too simple to expect splitting of the B 1s excitation into two excited states in the delocalized core picture, as opposed to splitting into four in the case of the localized core picture. In the localized core hole picture (which gives incorrect state symmetries) a compact description is possible and a single excited configuration is a good approximation. On the other hand, the delocalized core hole picture gives correct state symmetries, but a complicated multielectron configuration description is necessary. Wave functions with the correct symmetry can be generated from the localized-core-hole calculations by taking appropriate linear combinations as has been described elsewhere. ${ }^{37}$ Table 4 emphasizes this point by showing that the B 1s excitation energies and oscillator strengths of paracarborane generated within the delocalized picture by an extensive, extended basis set SCF-CI calculation are very similar to those calculated using a localized-core hole and a more modest basis set.

In $D_{5 d}$ symmetry only excitations from core orbitals with ungerade symmetry are dipole-allowed. These orbitals have $a_{2 u}$, $\mathrm{e}_{1 \mathrm{u}}$, and $\mathrm{e}_{2 \mathrm{u}}$ symmetries. Therefore, we have to consider excitations from these five core orbitals to the $\mathrm{e}_{1 \mathrm{~g}} *$ and $\mathrm{e}_{2 \mathrm{~g}}$ * unoccupied orbitals. The ground state is $\mathrm{A}_{1 \mathrm{~g}}$ and excited states with $A_{2 u}$ and $E_{1 u}$ are allowed, but there is no $B$ 1s excited state with $A_{2 u}$ symmetry. The electron configurations arising from the following $B$ 1s excitations correspond to states with $E_{1 u}$ (a)

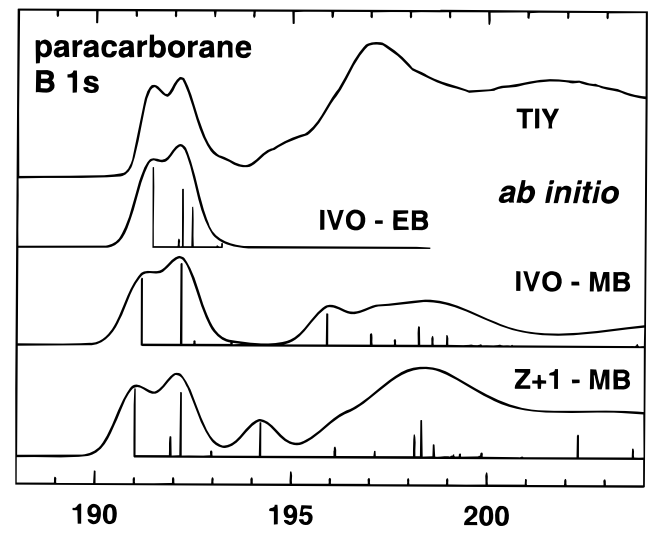

(b)

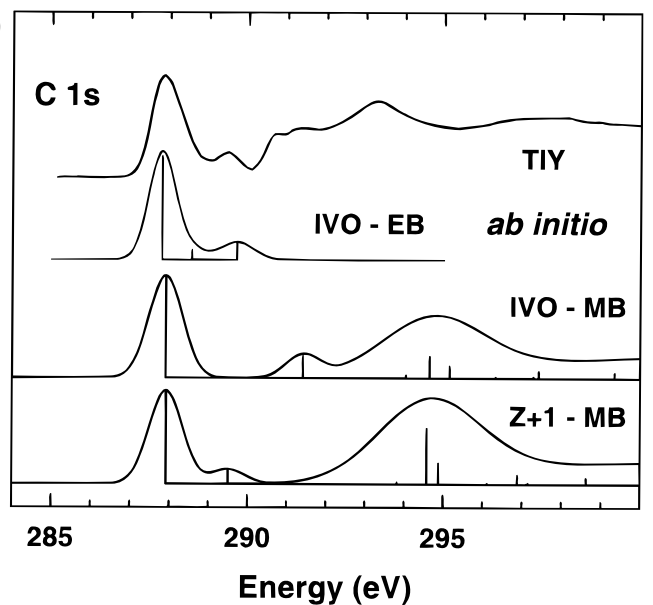

Figure 3. $\mathrm{B} 1 \mathrm{~s}$ and $\mathrm{C} 1 \mathrm{~s}$ spectra of paracarborane predicted from $\mathrm{ab}$ initio calculations compared to the high-resolution experimental TIY spectra: IVO = improved virtual orbital; $\mathrm{EB}=$ extended basis; $\mathrm{MB}$ $=$ minimum basis. The vertical lines indicate the energies and relative intensities of the spectral components that have been Gaussian broadened and summed to get the predicted spectra. The ab initio results were aligned at the first peak in each spectrum, which involved shifts of $-2.0,+2.0$, and $+1.4 \mathrm{eV}$ for the IVO-EB, IVO-MB, and $(Z+1)$ MB B 1s results.

symmetry:

$$
\begin{aligned}
& \mathrm{a}_{2 \mathrm{u}} \rightarrow \mathrm{e}_{1 \mathrm{~g}} \\
& \mathrm{e}_{2 \mathrm{u}} \rightarrow \mathrm{e}_{1 \mathrm{~g}} \\
& \mathrm{e}_{1 \mathrm{u}} \rightarrow \mathrm{e}_{2 \mathrm{~g}} \\
& \mathrm{e}_{2 \mathrm{u}} \rightarrow \mathrm{e}_{2 \mathrm{~g}}
\end{aligned}
$$

Among them, the first two configurations are nearly degenerate and the second two are nearly degenerate. However, we have to consider configuration mixing within $\mathrm{E}_{1 \mathrm{u}}$ symmetry. Thus, the first two configurations are strongly mixed and result in states that correspond to the first and third excited states in the localized picture. Similarly, the second two configurations mix strongly and result in states similar to the second and fourth excited states in the localized picture. The state splitting in the delocalized picture can be related to the orbital splitting in the localized picture, where configuration mixing among the localized core-hole configurations is very small.

3.4. Isomeric Carboranes: Interpretation of the $B$ 1s Spectra. After using paracarborane to explore the dependence of the ab initio results on the methodology, we have chosen to carry out calculations on $\mathrm{B} 1 \mathrm{~s}$ and $\mathrm{C} 1 \mathrm{~s}$ excitation in all three isomers using two techniques: the ab initio IVO-MB method with a localized-core hole and the semiempirical EHMOEICVOM method. Figure 5 presents a level diagram based on 
(a) Ground state

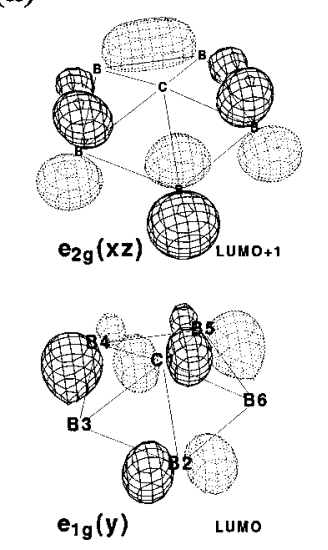

(b) C 1s excited states

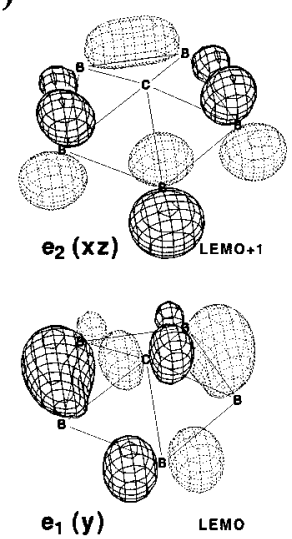

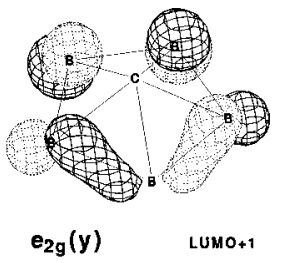
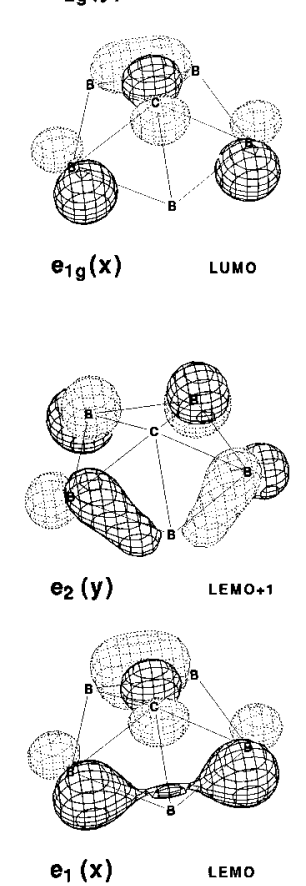

(c) B 1 s excited states

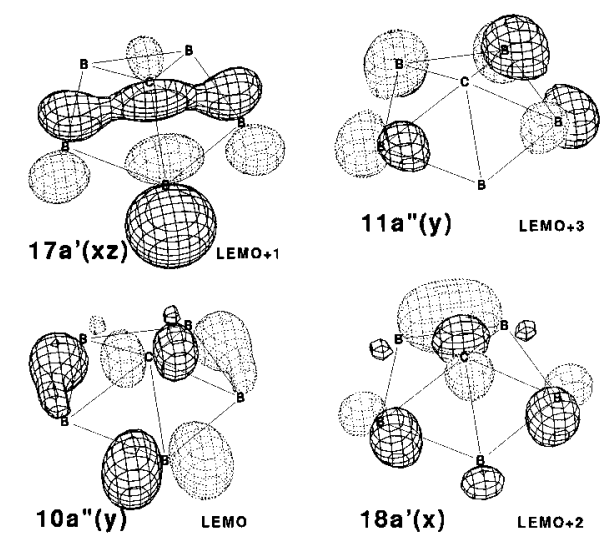

(d)

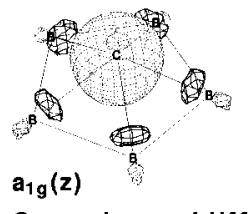

Ground state LUMO+2
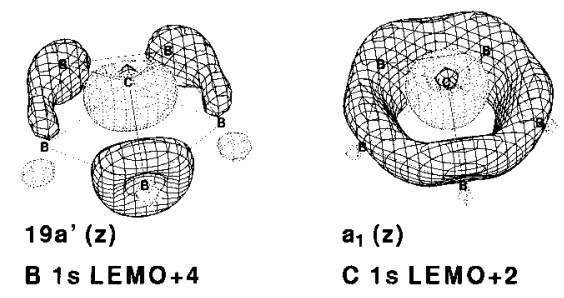

Figure 4. Plots of the four lowest unoccupied MOs of paracarborane: (a) the ground state; (b) the C 1s excited states; (c) the B 1s excited states, as predicted by the localized-core-hole minimum basis IVO ab initio calculations. Part d compares the next higher energy unoccupied orbital in the ground, B 1s, and C 1s excited states of paracarborane. For clarity only the contributions in the top half of the molecule are plotted, with the bottom half being identical. The orbital labeling refers to the reduced symmetry of $C_{5 v}$ in the $\mathrm{C} 1 \mathrm{~s}$ and $C_{s}$ in the B $1 \mathrm{~s}$ excited state within the localized core-hole approximation. These states are similar to the symmetry-adapted functions predicted in a delocalized, configuration interaction calculation (see text for further discussion of the localized versus the delocalized pictures).

TABLE 4: Energies and Oscillator Strengths for B 1s Excited closo-1,12-Paracarborane Predicted from High-Level ab Initio Calculations Using Localized and Delocalized Core Holes

\begin{tabular}{cccc}
\hline \multicolumn{3}{c}{ A. Localized Results } \\
\cline { 1 - 1 } ab initio & $\begin{array}{c}\text { extended Hückel } \\
\text { eigenvalue }\end{array}$ \\
\hline $10 \mathrm{a}^{\prime \prime}(y)$ & term value $(\mathrm{eV})$ & oscillator strength & 5.56 \\
$17 \mathrm{a}^{\prime}(x z)$ & 5.22 & 0.174 & 4.88 \\
$18 \mathrm{a}^{\prime}(x)$ & 4.88 & 0.213 & 4.42 \\
$11 \mathrm{a}^{\prime \prime}(y)$ & 3.94 & 0.0094 & 3.53 \\
\multicolumn{3}{c}{ B. Delocalized Results } \\
\hline \multicolumn{3}{c}{ ab initio } \\
\hline state & term value (eV) & oscillator strength \\
\hline $\mathrm{e}_{\mathrm{gg}}(y)$ & $5.46(2)^{a}$ & 0.2270 \\
$\mathrm{e}_{2 \mathrm{~g}}(x z)$ & $4.68(2)$ & 0.2050 \\
$\mathrm{e}_{\mathrm{g}}(x)$ & $4.48(1)$ & 0.0013 \\
$\mathrm{e}_{2 \mathrm{~g}}(y)$ & $4.21(1)$ & 0.0450
\end{tabular}

${ }^{a}$ In each case several transitions (2-6) were generated by the calculation at very similar energies. The mean energy, the standard deviation in energy, and the sum of the oscillator strengths are reported.

the EHMO energies of the low-lying unoccupied orbitals in each isomer. In each case, the diagram indicates correlation among orbitals that have a similar spatial distribution in the ground state, C 1s excited state, and each B 1s excited site. The levels that are assigned as similar are based on the orbital character predicted by the EHMO calculation. The orbital correlation picture is oversimplified because there is considerable orbital mixing in certain cases. Relative to the ground state, there is often $1-2 \mathrm{eV}$ stabilization when a core hole is introduced. The magnitude of this stabilization can be related to the electron density on the core excited atom in that orbital, as well as the extent of delocalization of the MO. Overall there appears to be very little isomeric dependence of the core hole stabilization or excitonic effects.

The B 1s spectra predicted by the IVO-MB calculations are presented in Figure 6 for each of the three isomers, in comparison with the experimental TIY spectrum. The B 1s spectra predicted by the EHMO calculation are presented in Figure 7. In each case the four distinct B 1s sites in orthocarborane and metacarborane have been taken into account using the calculated IPs for the ab initio and the experimental IPs for the EHMO results. To fully appreciate the nature of the core excitation, it is useful to examine the density distribution of each of the excited orbitals. However, space limitations preclude their full presentation. The IVO-MB orbitals of orthoand metacarborane are similar but not identical with those for paracarborane, which are plotted in Figure 4. In particular, there is a remarkable degree of similarity of the lowest unoccupied MOs of all three molecules, and thus, the MO plots in Figure 4 can be used to assist discussion as a first approximation. Essentially, the LEMO has a character at the core hole that is mainly that of a p orbital oriented tangentially to the surface of the compound. In the (LEMO +1$)$ orbital the $p$ component at the core-hole site is oriented perpendicular to the surface of the compound. The $(\mathrm{LEMO}+1)$ has more s character than the LEMO. 

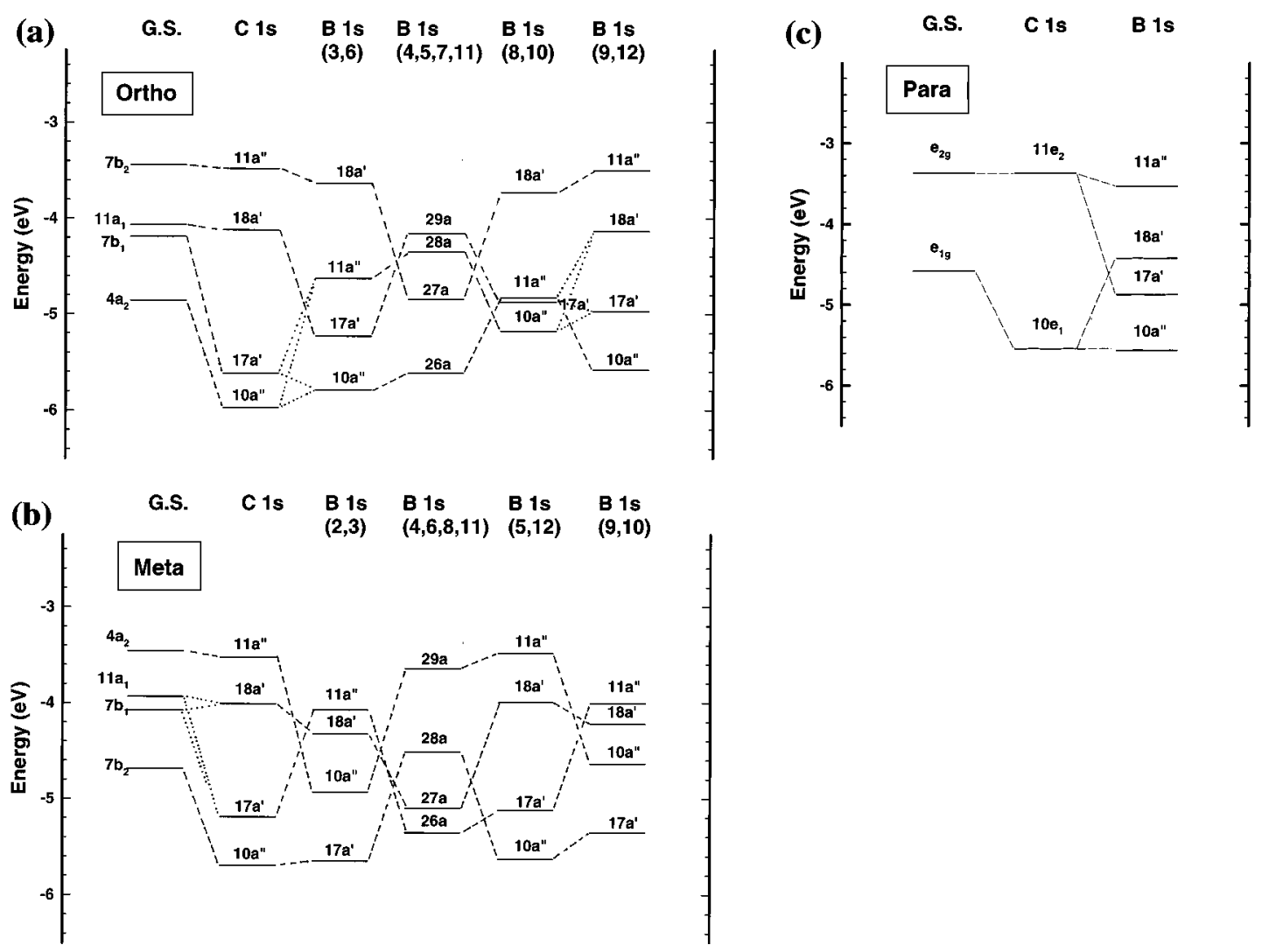

Figure 5. Orbital energy diagrams for the lowest four orbitals of the ground, C 1s, and B 1s excited states of (a) ortho-, (b) meta-, and (c) paracarborane as predicted by EHMO-EICVOM calculations. Dashed lines link orbitals of similar spatial character. Dotted lines indicate cases where there is considerable orbital mixing in one system relative to another.

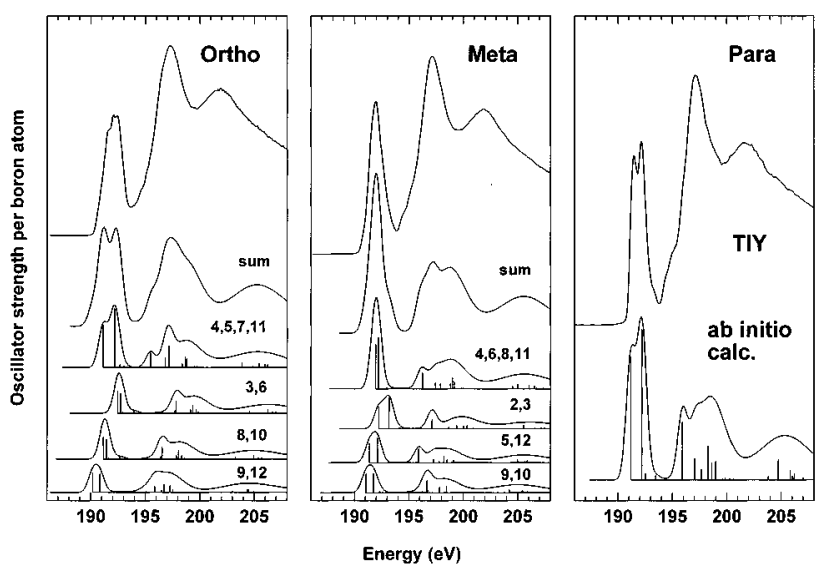

Figure 6. Comparison of calculated and experimental (high-resolution TIY) B 1s spectra of ortho-, meta-, and paracarborane. The calculated spectra are based on the ab initio IVO-MB results. The four components of the ortho and meta species are indicated. The predicted spectrum for each component was aligned at the appropriate IP, the components summed, and then the sum was rigidly shifted to obtain the best match to the first peak (average shift of $+1.8 \mathrm{eV}$ ).

The B 1s ionization potentials (IPs) of ortho- and metacarborane have been measured by X-ray photoelectron spectroscopy $\left(\mathrm{XPS}^{38}\right)$. The B 1s IP of the para isomer is assumed to be 195.6 $\mathrm{eV}$, the same as that of sites in orthocarborane and metacarborane where boron is directly attached to one carbon and four boron atoms. There is only a single B 1s IP in paracarborane, since all boron centers are chemically equivalent. If the relatively sparse spectral structure in the core spectra of these species was associated solely with overlaps caused by core level chemical shifts, paracarborane should have a better defined core excitation spectrum than the other two isomers. This would be the case for those transitions with almost constant term values, such as core $\rightarrow$ Rydberg transitions. However, core $\rightarrow$
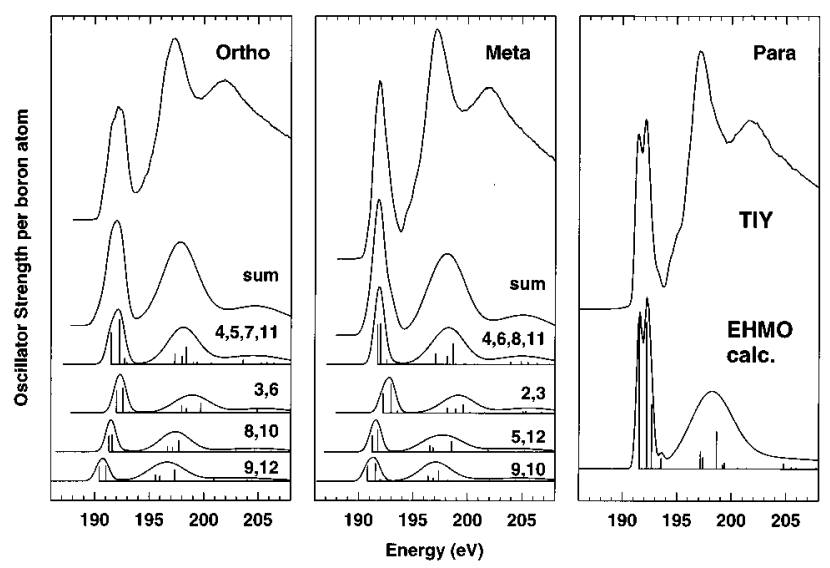

Figure 7. Comparison of EHMO predicted and experimental (highresolution TIY) B 1s spectra of ortho-, meta-, and paracarborane. The four individual components of the ortho and meta species are indicated. The predicted spectrum for each component was aligned at the appropriate IP, the components summed, and then the sum was rigidly shifted by $1.5 \mathrm{eV}$.

Rydberg transitions are generally much less intense than core $\rightarrow$ valence transitions, particularly in large molecules. Thus, we do not expect much correlation between the degree of complexity of XPS spectra and that of the B 1s excitation spectra. Indeed, experimentally, the sharpest B 1s excitation feature occurs in metacarborane. In this series of molecules it appears that the energies of the virtual orbitals, their dipole coupling to the ground state, and core level chemical shifts must all be taken into account in determining the B 1s spectral structure. The hypothesis of Green ${ }^{20}$ that the existence of four inequivalent boron atoms in orthocarborane might lead to four discrete structures is an oversimplification.

The low-energy region of the B 1s spectra is rather congested because there are several B 1s IPs with a spread of almost 2 
$\mathrm{eV}$ in orthocarborane and metacarborane and because there are a number of relatively closely spaced low-lying unoccupied orbitals. This is why there is relatively little change in any of these spectra on improving the spectral resolution from 0.7 to $<0.1 \mathrm{eV}$. However, the improved spectral resolution of the TIY spectra does reveal more spectral features than detected in the lower resolution ISEELS work. Although the higher resolution spectra do exhibit well-defined shoulders on the $192 \mathrm{eV}$ peak of paracarborane and orthocarborane, the lowest energy signal in metacarborane, which is the narrowest of the three, is virtually unchanged with improved resolution. The ab initio calculations (Figure 6) suggest that the lack of additional structure at higher energy resolution in the meta isomer may be the result of a large number of closely spaced transitions arising from the various symmetry inequivalent boron sites. Indeed, while the overall peak width (fwhm) is the smallest, the $192 \mathrm{eV}$ peak in metacarborane has a relatively wide base, consistent with contributions from a number of unresolved transitions.

The fine details of the discrete peak structure in the B 1s spectra of the three isomers are nicely reproduced in the ab initio (Figure 6) and EHMO (Figure 7) calculations. The shapes of corresponding components for each isomer are rather similar in both calculations. There is relatively little dependence on the B 1s site, although the spectrum of the 4-fold degenerate site is consistently different from that of the 2-fold degenerate sites. The largest change among the component spectra arises from the B 1s chemical shift, which is dependent on the proximity of the boron to carbon atoms. The carbons act as "electron-rich" centers that add density to adjacent boron atoms, shifting their IPs to lower energy.

The orbital correspondence in the B 1s excited states of orthocarborane and metacarborane is very complicated, not uniquely defined, and changes depending on which B 1s atom is excited, as shown in Figure 5. This reflects the significant delocalization that exists in the excited-state orbitals. However, the basic MO character is similar to that of the B 1s excited orbitals of paracarborane (Figure 4c). The lowest two orbitals, LEMO and (LEMO + 1), have p character oriented tangentially to the surface of the compound, and the excitations to LEMO and $(\mathrm{LEMO}+1)$ contribute to the main low-energy absorption peak as shown in Figure 6. Excitations to (LEMO +2$)$ and $(\mathrm{LEMO}+3)$ are predicted to produce a weak shoulder structure on the main B 1s excitation peak. The (LEMO + 4) orbital has sp hybrid character normal to the surface of the cage compound. A small peak around the ionization threshold is assigned to excitation to (LEMO + 4) as well as to Rydberg transitions.

Our ab initio calculations may be compared to those for B $1 \mathrm{~s}$ excited orthocarborane reported by Green et al. ${ }^{20}$ When the core-hole chemical shifts are incorporated, their results predict a five-line spectrum for orthocarborane that would be detected as two distinguishable peaks, the lower one being a single transition at $190.9 \mathrm{eV}$ with a relative intensity of 0.23 and the higher energy one at $191.9 \mathrm{eV}$ composed of three transitions with a total relative intensity of 0.91 . The predicted separation of $1 \mathrm{eV}$ in this calculation is similar to that between the two main components of the $192 \mathrm{eV}$ peak in orthocarborane (191.3 and $192.1 \mathrm{eV})$. Thus, there is reasonable agreement between both ab initio calculations and experimental results, in both the separation and the relative intensities of the components of the lowest energy band in the B 1s spectrum.

The present EHMO results for orthocarborane are very similar to those that were published earlier, ${ }^{17}$ with minor differences in predicted intensities probably being associated with small differences in the geometry used. The spectral simulations (Figure 6 (B 1s) and Figure 12 (C 1s) of ref 17) differ from the present result mostly because of a different choice of Gaussian

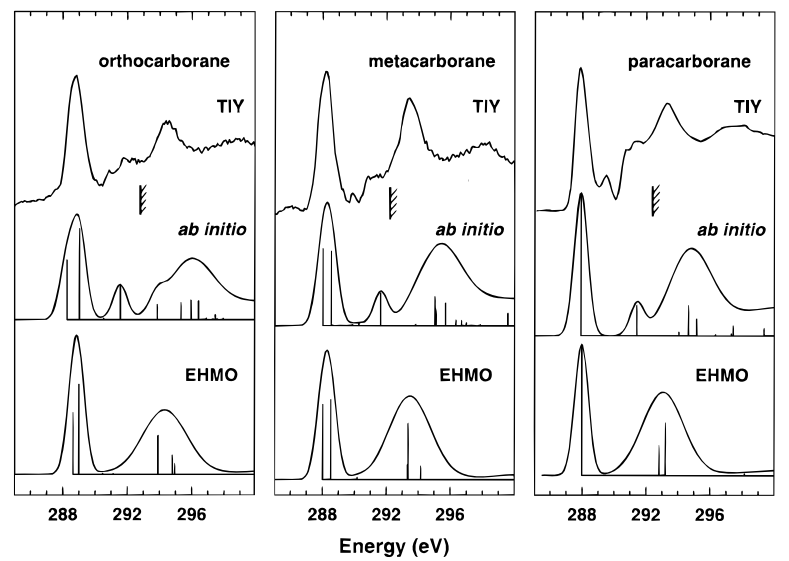

Figure 8. Comparison of ab initio (IVO-MB) and EHMO predicted spectra with the experimental (high-resolution TIY) C 1s TIY spectra of ortho-, meta-, and paracarborane. A $1.8 \mathrm{eV}$ rigid shift (the same for all isomers) was used for the ab initio while a $1.5 \mathrm{eV}$ rigid shift was used for the EHMO result.

line broadening. As was concluded earlier, the extended Hückel treatment gives much superior results to MNDO. Clearly, the $\mathrm{ab}$ initio results presented in this work are more reliable. Even so, EHMO-EICVOM has an impressive capability to reproduce the main core excitation spectral features. Although the relevance of EHMO-EICVOM has been demonstrated through comparison to experimental results for organic ${ }^{39,40}$ and organometallic ${ }^{41,42}$ species that have "conventional" two-center bonding, it is noteworthy that the present comparison to the ab initio results indicates EHMO is also a reasonable tool to assist spectral interpretation in extensively delocalized systems.

3.5. Carbon 1s Spectra. Figure 8 compares the experimental TIY spectra of each carborane with the C 1s spectra predicted by IVO-MB ab initio and EHMO calculations. As with the B 1s results, the predicted spectra are aligned at the energy of the first peak, resulting in shifts on the order of 1.5$2.0 \mathrm{eV}$ relative to the calculated energies (IP $+\mathrm{TV}$, where TV $=$ eigenvalue in the case of EHMO). The agreement between experiment and theory is reasonable but less good than for the corresponding B 1s spectra. In particular, neither calculation reproduces the detail in the experimental spectra between 290 and $293 \mathrm{eV}$. This may correspond to Rydberg excitations that cannot be represented by either minimal basis set calculation. Another problem is that peak overlap in the IVO-MB calculation predicts a systematic broadening of the first peak from 1.1 to $1.9 \mathrm{eV}$ in the order, para $\rightarrow$ meta $\rightarrow$ ortho, whereas the variation in the experimental peak width is much less-the peak widths are $0.9,1.0$, and $1.1 \mathrm{eV}$ for the para, meta, and ortho isomer, respectively.

The C 1s IPs of orthocarborane and metacarborane have been measured by XPS, ${ }^{38}$ while that of paracarborane has been estimated. If one uses the relative energies of the center of the lowest energy C 1s feature, this would suggest a C 1s IP of $291.8 \mathrm{eV}$ based on extrapolation from the IPs of orthocarborane $(292.8 \mathrm{eV})$ and metacarborane $(292.2 \mathrm{eV})$. However, both calculations clearly indicate that there are two components to the lowest energy excitation band in orthocarborane and metacarborane, whereas there is only a single transition in paracarborane, that to the lower energy of the pairs expected in ortho and metacarborane. Armed with this understanding, we note that the main peak in paracarborane aligns closely with the lower energy component of the main peak in metacarborane (see insert to Figure 2), which is reasonable, since the higher energy $\mathrm{C} 1 \mathrm{~s} \rightarrow \mathrm{e}_{2 \mathrm{~g}}$ transition is dipole forbidden in paracarborane. Based on this, we estimate the IP of paracarborane to be $292.2 \mathrm{eV}$, the same as that of metacarborane. The Mulliken population analysis of the charges on the carbon atoms based 
on the ab initio calculations supports this interpretation. It predicts charges of $-0.570,-0.581$, and -0.497 e for para, meta, and orthocarborane, respectively. The close similarity of the charge on carbon in the meta and para isomers suggests that their C 1s IPs will be similar. The higher C 1s IP of orthocarborane is consistent with it having a carbanion character relatively weaker than the carbon atoms in the other two isomers.

The energy of the $\mathrm{C} 1 \mathrm{~s} \rightarrow$ LEMO transition is relatively high compared, for example, to $\mathrm{C} 1 \mathrm{~s} \rightarrow \pi^{*}$ excitations in unsaturated organic compounds. One way of rationalizing this would be to consider that carbon in the carboranes has a partial carboniumlike character. ${ }^{43}$ However, that is inconsistent with the net charge transfer from B to $\mathrm{C}$ predicted by the Mulliken analysis. As the MO plots for paracarborane indicate (Figure $4 \mathrm{~b}$ ), the LEMO (of $\mathrm{e}_{1 \mathrm{~g}}$ symmetry in the para isomer) has a local " $\mathrm{p}-$ $\pi^{*}$ " character in that it is composed of two orthogonal C $2 p$ orbitals at the carbon atoms. One might consider this as supporting the occurrence of a localized $\mathrm{C}=\mathrm{C}$ bond in the ortho isomer. ${ }^{39}$ However, this is incorrect, since the LEMO has this " $\mathrm{p}-\pi^{*}$ " character at both carbon and boron sites in all three isomers.

The ab initio calculations indicate that the sharp C 1s peak in all three isomers arises from excitation to orbitals that have the $\mathrm{C} 2 \mathrm{p}$ component at the core excited atom oriented along the faces of the carborane cages. Within the localized corehole picture, the $\mathrm{C} 1 \mathrm{~s}$ excited states of ortho- and metacarborane belong to the same symmetry $\left(C_{2 v}\right)$ as the B 1 s excited states of paracarborane. In fact, the orbitals of $\mathrm{C} 1 \mathrm{~s}$ excited orthoand metacarborane are nearly the same as those of B 1s excited paracarborane. The degeneracy in the lowest excited orbitals that exists in paracarborane is removed in ortho- and metacarborane, but the energy splitting is not as large as in the B 1s excited states of paracarborane. That is, the first main peak in the $\mathrm{C} 1 \mathrm{~s}$ spectra of ortho- and metacarborane arises from excitations to the lowest orbitals, which are related to the $\mathrm{e}_{1 \mathrm{~g}}$ (y) and $\mathrm{e}_{1 \mathrm{~g}}(x)$ orbitals in $D_{5 d}$ symmetry (Figure $4 \mathrm{a}$ ). Excitations to the second lowest energy orbitals, which are related to the $\mathrm{e}_{2 \mathrm{~g}}(x z)$ and $\mathrm{e}_{2 \mathrm{~g}}(y)$ orbitals of paracarborane, are now dipole allowed in the reduced symmetry of ortho- and metacarborane. They are predicted to produce substructure (splitting or a shoulder) on the main peaks. Features between the main peak and the ionization threshold arise from Rydberg states as well as excitation to the $\mathrm{a}_{1}(z)$ orbital. The latter orbital is sketched in Figure 4d.

Experimentally, paracarborane has the sharpest main $\mathrm{C} 1 \mathrm{~s}$ feature, consistent with the symmetry forbidden character of the $\mathrm{C} 1 \mathrm{~s} \rightarrow \mathrm{LEMO}+1$ transition, as discussed in detail earlier. In the meta and ortho isomers both the ab initio and EHMO calculations indicate that there should be two components, as just outlined. However, a splitting associated with these components is not resolved in the $\mathrm{C} 1 \mathrm{~s}$ spectra, although the first peak in the $\mathrm{C} 1 \mathrm{~s}$ spectrum of the ortho isomer is broader and has a low-energy shoulder while there is a clear low-energy shoulder to the main peak of the $\mathrm{C} 1 \mathrm{~s}$ spectrum of metacarborane (these details are best seen in the insert to Figure 2).

3.6. Quantitative Comparison of TIY and Absorption Spectra. Figure 9 compares the TIY spectrum of orthocarborane in the $\mathrm{B} 1 \mathrm{~s}$ and $\mathrm{C} 1 \mathrm{~s}$ regions to the corresponding absorption spectrum derived from the energy loss spectrum by conversion via the Bethe-Born kinematic factor. ${ }^{23}$ The signalto-background ratio and relative intensities of absorption and TIY are similar for the other two isomers. The ratio of the TIY to the absorption ("abs") curve is plotted in the upper sections of parts a and b of Figure 9 for all three isomers (note the offsets). This ratio is a measure of the ionization efficiency ( $\eta$, the number of ions per absorbed photon). The TIY/"abs" ratio increases considerably in the region of the $\mathrm{B} 1 \mathrm{~s}$ ionization
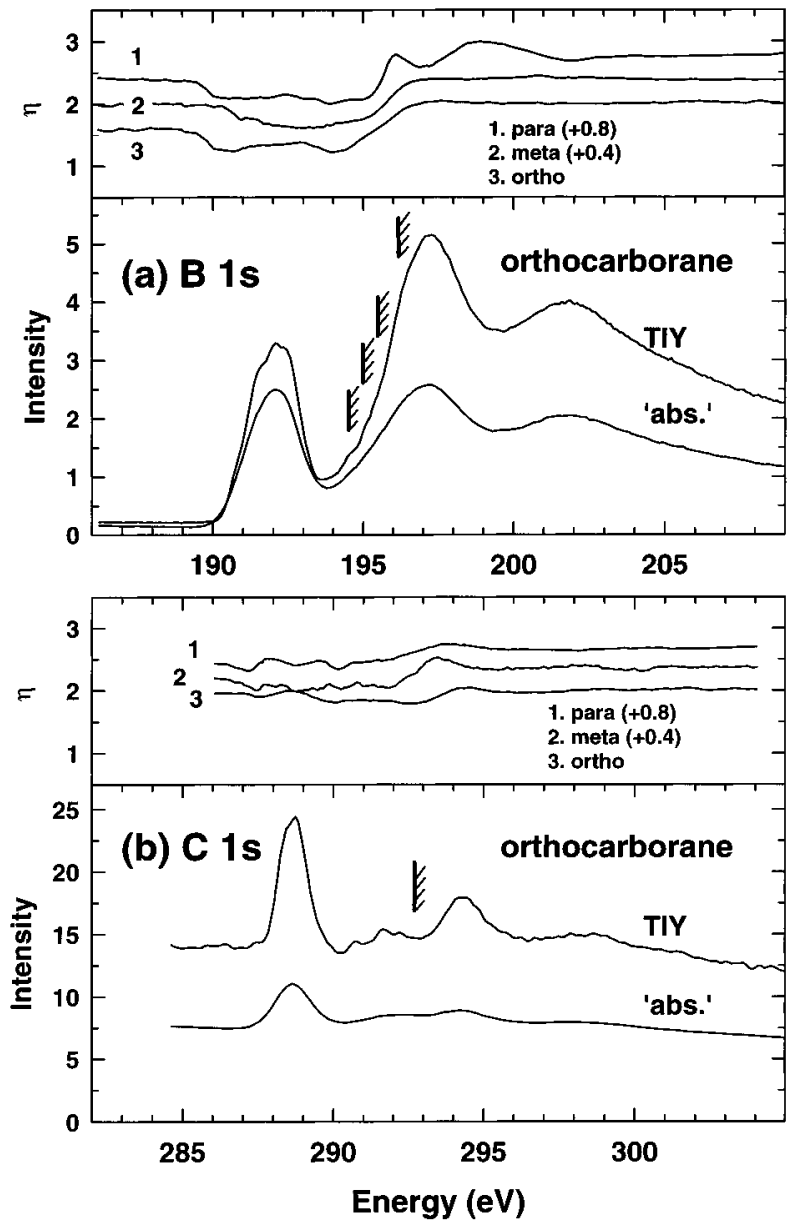

Figure 9. Comparison of the absorption (derived from electron energy loss) and total ion yield spectra of orthocarborane in (a) the B $1 \mathrm{~s}$ and (b) the C 1s regions. The TIY and "abs" curves have been scaled so that the ratio is approximately 2 in the core ionization continua. The ratio of the TIY and "abs" signals, which is an estimate of the ionization efficiency, is plotted in the upper panel of parts $a$ and $b$ for all three isomeric carboranes. An offset of 0.4 and 0.8 has been applied to the metacarborane and paracarborane curves to avoid overlap.

energies. The rise at the continuum threshold is sharpest for paracarborane, consistent with the single IP of this species. Both observations are consistent with interpreting this ratio as a measure of $\eta$. The relative spectral intensities have been adjusted such that $\eta$ is approximately 2 in the B $1 \mathrm{~s}$ and $\mathrm{C} 1 \mathrm{~s}$ core ionization regions. This is a typical value of $\eta$ for core ionization of atoms and molecules in the soft X-ray regime, since the Auger decay produces a doubly charged ion that dissociates into two (or more) singly charged ions in most events. The detailed origin of $\eta$ in terms of individual fragmentation processes throughout the $\mathrm{B} 1 \mathrm{~s}$ and $\mathrm{C} 1 \mathrm{~s}$ regions is the subject of the accompanying paper. ${ }^{16}$

The same spectral features are detected in the TIY and absorption spectra, but the spectral intensities vary systematically. $\eta$ is relatively depressed in the region of the discrete $192 \mathrm{eV}$ feature, indicating that there is a reduced amount of double ionization from the discrete core excited states. This corresponds to producing singly ionized final states after resonant excitation. When the core electron is not involved (spectator) or if it partakes (participator) in the electronic Auger decay, only one electron gets ejected. The rather slow rise in $\eta$ for ortho- and metacarborane in the region of the core ionization onset is related in part to the spread in B 1s IPs for different sites and in part to postcollision interaction effects. The variation in $\eta$ is smaller in the $\mathrm{C} 1 \mathrm{~s}$ regime (lower panel of Figure 9), with $\eta$ being modulated only between 1.8 and 2 . The $\mathrm{C}$ 1s edge in carboranes has little influence on the overall 
ionization efficiency because $\mathrm{C} 1$ s excitation is a relatively small portion of the total absorption. Surprisingly, $\eta$ increases rather than decreases at the $\mathrm{C} 1 \mathrm{~s} \rightarrow$ LEMO transition. This might be an artifact associated with different resolution in the TIY and "abs" measurements or, alternatively, it could reflect increased dissociative double ionization. As expected, there is a slight increase in $\eta$ near the $\mathrm{C} 1 \mathrm{~s}$ ionization threshold.

The motivation of this study was to provide useful information concerning the mechanisms of photofragmentation that might help to understand the use of photodissociation as a means to generate high-quality boron carbide films from these materials. The high levels of fragmentation that are documented in the accompanying paper ${ }^{16}$ indicate that Coulomb explosion following core-hole creation in these species is a very effective means of creating fragments. The B 1 s and C 1s spectra analyzed in this work provide a means to rationalize the photon energy dependence of the fragmentation.

\section{Summary}

This study has provided new spectral data for core excitation in a series of isomeric carborane compounds. The spectra of the three isomeric carboranes are relatively similar, with the main variations being energy shifts in the $\mathrm{C}$ 1s region chiefly associated with core level chemical shifts, as well as changes in the detailed line shape of the lowest energy B 1s peak and the weak structure around $290-292 \mathrm{eV}$ in the C 1s spectra. The reduced symmetry and multiple $\mathrm{B} 1 \mathrm{~s}$ environments in orthoand metacarborane, relative to paracarborane, are key factors in determining the line shapes. The B 1s and C 1s spectral features have been assigned with the aid of interspecies comparisons and through comparisons to both ab initio and extended Hückel calculations. The comparison of ab initio and EHMO results indicates that the simple EHMO-EICVOM approach can be quite useful for assisting spectral interpretation, not only in "conventional" complexes (those with two-center bonding) but also in molecules that have no simple two-center bonding model and for which the electronic structure is extensively delocalized.

Acknowledgment. This work was financially supported by the Natural Sciences and Engineering Research Council of Canada, the National Science Foundation (Grant No. DMRINT-9300238), NATO, and the W. M. Keck Centre for Molecular Electronics. A.P.H. thanks the Lawrence Berkeley National Laboratory for its support and hospitality during a research leave. The Advanced Light Source is funded by the Department of Energy. We thank the staff of the Advanced Light Source for their assistance and expert operation of the facility. Contributions to the data acquisition were made by Sunwoo Lee and Dongjin Byun, while assistance with synthesis was provided by John A. Glass, Jr.

\section{References and Notes}

(1) (a) Lee, S.; Mazurowski, J.; Ramseyer, G.; Dowben, P. A. J. Appl. Phys. 1992, 72, 4925. (b) Mazurowski, J.; Baral-Tosh, S.; Ramseyer, G.; Spencer, J. T.; Kim, Y-.G.; Dowben, P. A. Mater. Res. Soc. Symp. Proc. 1991, 190, 101. (c) Mazurowski, J.; Lee, Sunwoo; Ramseyer, G.; Dowben, P. A.; Mater. Res. Soc. Symp. Proc. 1992, 242, 637.

(2) (a) Lee, S.; Dowben, P. A. Appl. Phys. 1994, A58, 223. (b) Lee, S.; Ton, T.; Zych, D.; Dowben, P. A. Mater. Res. Soc. Sym. Proc. 1993, $283,483$.

(3) Byun, D.; Hwang, S.-D.; Dowben, P. A.; Perkins, F. K.; Filips, F.; Ianno, N. J. Appl. Phys. Lett. 1994, 64, 1968.

(4) Hwang, S.-D.; Byun, D.; Ianno, N. J.; Dowben, P. A.; Kim, H. R. Appl. Phys. Lett. 1996, 68, 1495.

(5) (a) Perkins, F. K.; Rosenberg, R. A.; Lee, Sunwoo; Dowben, P. A. J. Appl. Phys. 1991, 69, 4103. (b) Rosenberg, R. A.; Frigo, S. P.; Lee, S.; Dowben, P. A. J. Appl. Phys. 1992, 71, 4795.
(6) (a) Avouris, Ph.; Lyo, I. W.; Bozzo, F.; Kaxiras, E. J. Vac. Sci. Technol. 1990, A8, 3405. (b) Avouris Ph. J. Phys. Chem. 1990, 94, 2246. (c) Lyo, I.-W.; Kaxiras, E.; Avouris, Ph. Phys. Rev. Lett. 1989, 63, 1261.

(7) Deutsch, T. F.; Fan, J. C. C.; Turner, G. W.; Chapman, R. L.; Ehrlich, D. J.; Osgood, R. M. Appl. Phys. Lett. 1981, 38, 144. 3275 .

(8) Huang, H.; Tong, S. Y.; Quinn, J.; Jona, F. Phys. Rev. B 1990, 41,

(9) McLean, A. B.; Terminello, L. J.; Himpsel, F. J. Phys. Rev. B 1990 41,7694 .

(10) Rowe, J. E.; Wertheim, G. K.; Riffe, D. M. J. Vac. Sci. Technol. A 1991, 9, 1020 .

(11) Ma, Y.; Rowe, J. E.; Chaban, E. E.; Chen, C. T.; Headrick, R. L.; Meigs, G. M.; Modesti, S.; Sette, F. Phys. Rev. Lett. 1990, 60, 2172.

(12) (a) Byun, D.; Spady, B. R.; Ianno, N. J.; Dowben, P. A. Nanostruct. Mater. 1995, 5, 465. (b) Byun, D.; Hwang, S.-D.; Zhang, J.; Zeng, H.; Perkins, F. K.; Vidali, G.; Dowben, P. A. Jpn. J. Appl. Phys. 1995, 34, L941.

(13) Zeng, H.; Byun, D.; Zhang, J.; Vidali, G.; Onellion, M.; Dowben, P. A. Surf. Sci. 1994, 313, 239.

(14) (a) Higashi, I. AIP Conf. Proc. 1986, 140, 1c. Lundstrum, T. Ibid. 1986, 140, 19. Yakel, H. L. Ibid. 1986, 140, 97. Clark, H. K.; Hoard, J. L. J. Am. Chem. Soc. 1943, 65, 2115. Will, G.; Kossobutzki, K. H. J. LessCommon Met. 1976, 44, 87. Larson, A. C.; Cromer, D. T. Acta Crystallogr. A 1972, 28, 553. Yakel, H. L. Acta Crystallogr. B 1975, 31, 1797. Kirfel, A.; Gupta, A.; Will, G. Acta Crystallogr. B 1979, 35, 1052; 1979, 35, 2291.

(15) Morosin, B.; Mullendore, A. W.; Emin, D.; Slack, G. A. AIP Conf. Proc. 1986, 140, 70. Will, G.; Kirfel, A. Ibid. 1986, 140, 87. Emin, D. Ibid. 1986, 140, 189.

(16) Hitchcock, A. P.; Kilcoyne, A. L. D.; Tyliszczak, T.; Rühl, E.; Bozek, J. D., Jr.; Spencer, J. T.; Dowben, P. A. J. Phys. Chem., to be submitted.

(17) Hitchcock, A. P.; Wen, A. T.; Lee, S.; Glass, J. A., Jr.; Spencer, J. T.; Dowben, P. A. J. Phys. Chem. 1993, 97, 8171

(18) Howard, I. A.; Beckel, C. L.; Emin, D. Phys. Rev. B 1987, 35, 9265.

(19) (a) Ruckman, M. W.; Murray, M. F.; Mowlem, J. K.; Moore, J. F.; Strongin, D. R. Chem. Phys. Lett. 1992, 199, 449. (b) Ruckman, M. W.; Murray, M. F.; Mowlem, J. K.; Strongin, D. R. J. Vac. Sci. Technol. A 1993, 11, 2477

(20) Green, T. A. In Boron-Rich Solids; Emin, D., Aselage, T. L., Switendick, A. C., Morosin, B., Eds.; AIP Conference Proceedings 231; American Institute of Physics: New York, 1991; p 42.

(21) Green, T. A.; Switendick, A. C.; Emin, D. J. Chem. Phys. 1988 89,6815 .

(22) Hitchcock, A. P. Phys. Scr., T 1990, 31, 159.

(23) Hitchcock, A. P.; Mancini, D. C. J. Electron Spectrosc. Relat. Phenom. 1994, 67, 1 .

(24) Langer, B.; Berrah, N.; Farhat, A.; Hemmers, O.; Bozek, J. D. Phys. Rev. A 1996, 53, R1946.

(25) Bohn, R. K.; Bohn, M. D. Inorg. Chem. 1971, 10, 350.

(26) Kosugi, N.; Kuroda, H. Chem. Phys. Lett. 1980, 74, 490.

(27) Huzinaga, S.; Andzelm, J.; Klobukowski, M.; Radzio-Andzelm, E.; Sasaki, Y.; Tatewaki, H. Gaussian Basis Sets for Molecular Calculations; Elsevier: Amsterdam, 1984.

(28) Schwarz, W. H. E. Chem. Phys. 1975, 11, 217.

(29) Hunt, W. J.; Goddard, W. A., III. Chem. Phys. Lett. 1969, 3, 414.

(30) Kosugi, N.; Shigemasa, E.; Yagishita, A. Chem. Phys. Lett. 1992, $190,481$.

(31) Kosugi, N.; Adachi, J.; Shigemasa, E.; Yagishita, A. J. Chem. Phys. 1992, 97, 8842 .

(32) Iwata, S.; Kosugi, N.; Nomura, O. Jpn. J. App. Phys. 1978, 17-S2, 109.

(33) Kosugi, N. Theor. Chim. Acta 1987, 72, 149.

(34) Meali, C.; Proserpio, D. M. J. Chem. Educ. 1990, 67, 399.

(35) Langhoff, P. W. In Methods in Computational Molecular Physics; Diercksen, G. H. F., Wilson, S., Eds.; Reidel: Dordrecht, 1983; p 299.

(36) Sheehy, J. A.; Gil, T. J.; Winstead, C. L.; Farren, R. E.; Langhoff, P. W. J. Chem. Phys. 1989, 91, 1796. Gil, T. J.; Winstead, C. L.; Sheehy, J. A.; Farren, R. E.; Langhoff, P. W. Phys. Scr., T 1990, 31, 179.

(37) Denis, A.; Langlet, J.; Malrieu, J. P. Theor. Chim. Acta 1975, 38, 49. Cederbaum, L. S.; Domcke, W. J. Chem. Phys. 1977, 66, 5084. Àgren, H.; Bagus, P. S.; Roos, B. O. Chem. Phys. Lett. 1981, 82, 505.

(38) Allison, D. C.; Johansson, G.; Allan, C. J.; Gelius, U.; Siegbahn, H.; Allison, J.; Siegbahn, K. J. Electron Spectrosc. Relat. Phenom. 1972, $1,269$.

(39) Francis, J. T.; Hitchcock, A. P. J. Phys. Chem. 1994, 98, 3650.

(40) Francis, J. T.; Hitchcock, A. P. J. Phys. Chem. 1992, 96, 6598

(41) Rühl, E.; Wen, A. T.; Hitchcock, A. P. J. Electron Spectrosc. Relat. Phenom. 1991, 57, 137.

(42) Wen, A. T.; Rühl, E.; Hitchcock, A. P. Organometallics 1992, 11 , 2559.

(43) (a) Hawthorne, M. F.; Berry, T. E.; Weigner, P. A. J. Am. Chem. Soc. 1965, 87, 4746. (b) Lipscomb, W. N. Ibid. 1966, 88, 628.

(44) Boer, F. P.; Strerib, W. E.; Lipscomb, W. N. Inorg. Chem. 1964, 13, 1666 . 